\title{
Publish or Perish
}

It gives me immense pleasure and pride to write the editorial for the annals of my beloved alma mater. Within the realms of this great institution, I spent the best years of my youth burning the midnight oil mastering the science and art of healing both the body and soul of ailing humanity and striving hard to diligently absorb the words and works of my teachers, all maestros in their fields. Coming so close to suffering and death on such a regular basis humbled me greatly; it also incited me to do more to alleviate the anguish of my fellow humans.

It is this "more" that I wish to stress upon, for I am sure every colleague in my field does his or her bit in maintaining the nobility and probity of our profession to the utmost. "More" in this context means progression and progression is analogous with research; exploring new horizons not yet ferreted out but carrying great potential to benefit and ensure the survival of our species. Research always stems from a curious mind and curiosity is inherent to the nature of mankind. Unfortunately, the version of pedagogypervasive in our system seems to fear curiosity. After all, it is supposed to have killed the proverbial cat, so instead, rote learning is promoted. I think both students and teachers even at the elementary level should be trained to segregate impudence from the innocuous and fruitful quest to delve into the unknown.

Just as the most avid readers go on to become the greatest writers, studying the work and research of others acts as a stimulus to do so ourselves. Making research journals available should be a priority of every institution. Moreover, research should be made an integral part of the curriculum in colleges and universities. Problem based and project based learning, especially in groups should be encouraged. Opinions sequestered from a multitude of minds encourage criti- cal and lateral thinking, providing a nidus for generating many new hypotheses, which of course require research to prove their validity.

With the world on hand at the click of a button, research today is more tangible than ever. I personally believe that being computer savvy will help as promoting and inculcating research as part of our culture. Since research is a prerequisite to the development of a country, monetary or other incentives can be quite effective in luring and encouraging both the researcher and his subjects, when the zest to instigate research is lacking per se.

However, within the precincts of research also lie innumerable dark secrets. Adolph Hitler was a great protagonist and promoter of research to satisfy his own whims and vices. To pursue and fulfill Hitler's fantasy of creating a perfect and superior Caucasian race, replete with blond hair and blue eyes, the infamous Nazi doctor, Josef Mengele carried out inhumane experiments on concentration camp prisoners, especially twins. That was research in its most hideous form; not to benefit humanity, but to filter it out.

Today universities all over the world are ranked according to their research statistics. As Arrivistes striving to enter the clique of internationally acclaimed institutions, we need to adopt the "The Publish or Perish" phenomenon rampant in the West. However, merely putting on a supercilious patina of carrying out research is not enough. Choosing the right topic relevant to the incumbent problems, communicating effectively to assert our opinions and inferences as well as selecting the right journal to publish it in, are important to promote and uphold our perspective. Only after it has proved its credibility will research be able to permeate the current system and alter it to the benefit of humanity.
Prof. Dr. Farid Ahmad Khan

FRCS (Ed.), FCPS (Plastic surgery) Prof. of Cosmetic Plastic Surg. Principal, Chairman and Dean Shaikh Zayed Medical Complex, Lahore 